systems in the areas where the research had been carried out, it was not always easy to use the same data for engineering purposes in different geographical regions. It is clearly not practicable always to make pilot investigations before every engineering application, and the aim must therefore be to achieve the best fundamental understanding of the propa- gation phenomena from those experiments which are made, and thus so far as possible to ease the task of extrapolation.

The papers presented at the symposium and the discussion are to be published in a special issue of the Proceedings of the Institution of Electrical Engineers $(105, \mathrm{~B} ; 1958)$.
J. A. SAXTON

\title{
MACHINES AS MODELS IN BIOLOGICAL EXPERIMENTS
}

TN May 1957 the Laboratory Animals Bureau 1 arranged a symposium on humane technique in the laboratory. In a paper now available separately*, Dr. W. Grey Walter showed how machines could be used as models in biological experiments.

Walter first showed how the classical method of demonstrating physiological laws and principles are derived from those worked out for the physical sciences. The essence of these methods is the isolation of a single variable and the observation of this variable in various experimental conditions. In order to achieve this situation with living creatures, some sort of preparation is required. This involves dissection, mutilation or anæsthesia, often all three, and in this way a whole animal can be transformed into a working model of some particular function. For example, the Sherringtonian approach to reflex action of the spinal cord depended upon decerebration of a complex animal such as a cat and the restriction of attention to one, or occasionally two, reflex circuits. The results obtained in this way form the basis for much thinking about the nervous system, but it is now realized that the general picture of central nervous action derived from these studies of isolation, though admirably clear, lacks perspective and detril when related to animal behaviour.

If it is accepted that animal preparations are essentially models of working hypotheses, it follows that such hypotheses may as legitimately be embodied in machines as in mutilated animals. The use of machines may even present certain advantages : the components of a mechanical system can be accurately enumerated and specified, and the principle of parsimony, so important to fertile thinking, can be adhered to without inconvenience. For example, it is difficult to prove that a spinal cord preparation contains only those components necessary for the reflex action under investigation, but it is quite simple to demonstrate that a reflexive machine contains only essential elements.

The principles to be observed in the construction and use of animel models may be summarized as

* Laboratory Animals Bureau. Collected Papers, Vol. 6, 1957. Pp. 81. (London: Laboratory Animals Bureau, M.R.C. Laboratories,
1957.) 10s. follows : (1) the system to be modelled should contain more than one variable; (2) the model must contain no redundant components, in order to maintain parsimony; (3) the behaviour of the model should exhibit more features of behaviour than were originally planned or foreseen. Applying these principles, a number of models of animal function and behaviour have been constructed and are in frequent use both for the design of fresh experiments and for teaching purposes. These are:

(1) NERISSA-A Nerve Excitation, Inhibition and Synaptic Analogue. This demonstrates particularly the relationship between the various parameters of nervous action such as finite propagation-rate, excitation threshold, all-or-none conduction, strengthduration curves of excitability, refractory periods, Wedensky inhibition, synaptic facilitation and inhibition, inhibitory escape and rebound, transmission or information by pulse-interval modulation, and anomalies of 'inhibition of inhibition' and 'inhibition of inhibition of inhibition' during rhythmic as opposed to sustained stimulation.

(2) IRMA - Innate Releaser Mechanism Analogue, demonstrating the exclusive action of an excitatory state maintained by a cascade of neuronic elements and the effect upon this condition of variations in excitatory level.

(3) ELMER -Electro-Mechanical Robot, containing two olements equivalent to synaptic junctions, with two receptors and two effectors, to demonstrate the complexity of behaviour arising from the interaction of two reflex circuits coupled to a complex environment. The behaviour patterns observable include goal seeking, obstacle avoidance, discrimination between immediate and remote goals, choice between two equivalent goals, tendency to seek optima rather then maxima and elements of self- and socialclassification.

(4) CORA-A Conditioned Reflex Analogue, demonstrating the action of selective and storage mechanisms in the establishment of a contingency association between signals on a basis of statistical significance.

Such models provide both student and experimenter with an opportunity to classify observations of real animals and to design crucial experiments.

\section{EDIBLE LIZARDS}

\footnotetext{
$\triangle$ LLAN R. HOLMBERG, of the Department of Sociology and Anthropology of Cornell University, has described how natives of the north coast of Peru capture small lizards called cañanes partly for their nutritive but especially for their curative and aphrodisiacal properties*.

* Fieldiana Anthropology, 36, No. 9. (Chicago Natural History
Museum, 3957.) 75 cents.
}

The lizards, Dicrodon hoombergi, are found in the Chao Valley and dig their holes in the desert sand around clusters of the guarango tree (Prosopis juliflora)—called algarrobo in Viru-the fruits of which constitute their sole means of subsistence. They are not gregarious, but hundreds of pairs of them make their homes around nourishing stands of algarrobo. The full-grown males are bluish in 